\title{
The Influence of Medical Diagnostic Imaging Learning Based on PACS Case Teaching System Opening
}

\author{
Ge Yanming, Zhang Gaorui, Zhu Hong, Tu Yingshan, Long Jinfeng, Wang Xizhen, Dong \\ Peng*
}

Medical Imaging Center of the Affiliated Hospital, Wei fang Medical University, Weifang, China geyanming2001@163.coml

*corresponding author: Dong Peng

Keywords: case based PACS teaching system; medical diagnostic imaging; teaching quality.

\begin{abstract}
Objective To investigate the advantage of teaching the medical diagnostic imaging by opening the case teaching system based on the picture archiving and communication systems (PACS). Methods: In the process of medical diagnostic imaging teaching in the medical university, let the students study independently in their spare time by open the case teaching system based on PACS (application models of PACS, multimedia classroom, huge screen projecting). Results: By using the case based PACS teaching system, the teaching methods and teaching content have been enriched. The students' learning interest was improved, and their evaluation of teaching satisfaction was enhanced. Conclusion: Opening the case teaching system based on PACS can help to improve the teaching quality of the medical diagnostic imaging.
\end{abstract}

\section{Introduction}

Medical diagnostic imaging is a subject using the medical images to display the internal organs (include the brain, skull, throat, lung, stomach, abdomen, kidney, etc), structures and even functional information of the human body, and to make a localization diagnosis and qualitative diagnosis of the lesions. With the rapid development of technology, department of Medical Imageology in the hospital plays more and more important roles in the clinical practice. As a highly practical clinical specialty, the study of medical diagnostic imaging is an important part of the daily learning of undergraduates who majored in medical imageology specialty. The purpose of the Medical Diagnostic Imaging is to train students' reading-film ability to meet the needs of clinical practice, which means that the students should make the correct diagnosis based on imaging images.

Therefore, the learning effectiveness of undergraduates who majored in medical imageology specialty has a large extent affect and even decide the undergraduates whether can get a job and their clinical level of business [1].

With the rapid development of medical equipment and the increasing in the amount of the patients, the clinicians pay more and more attentions to medical imageing examination year by year. Under this background, the demand for the professionals of medical imageology specialty is increasing. Many department of Medical Imageology in the hospitals have higher requirement on the medical imaging undergraduate on their clinical skill level.

A series of breakthroughs in high-speed computing equipment, network communication and image processing technology in hospitals provide a realistic digital technology foundation for the digital acquisition, storage, management, processing, transmission and effective use of medical images. Digital imaging technology, modern communications and computer technology consist of the PACS (picture archiving and communication systems) system. The PACS system has become popular in many hospitals in China.

From the early 21st century, we built the intramural teaching PACS system [2], and use it in the theory of medical diagnostic imaging and experimental teaching. After several years of practice, the successful establishment of the Case based PACS teaching system provides an advanced means for the teaching of Medical diagnostic imaging, and we have achieved good effects in the experimental 
teaching process. According to open the Case based on PACS teaching system, this study discuss deeply about its role in the learning of the Medical diagnostic imaging for the undergraduates.

\section{Materials and Methods}

\subsection{Teaching Objects and Teaching Status Quo}

In the medical diagnostic imaging, the theoretical and experimental teaching of the undergraduate is one of the main teaching works to undertake of our department. Since the beginning of the 21st century, the PACS system has been established on the basis of the original digital medical equipment, realizing all-digital and film-free operation for more than 10 years in our department. The department undertakes the imaging medical teaching for over 60 years. We open the laboratory of the case based PACS teaching system every afternoon from Monday to Friday, students majored in medical imageology specialty who authorized by the laboratory can research cases in the computer terminal access and share teaching resources together.

\subsection{PACS Basic Equipment and Configuration}

The case based PACS teaching system is consisted of intramural teaching PACS network and video multimedia classrooms. PACS case-based teaching system is based on intramural equipment status and the construction of the local area network construction of the teaching system. Hardware components: one server and two sets of multimedia projection systems, the standard configuration of 120 computers and so on. Students can use the login account to read image and post-processing functions (Figure 1).

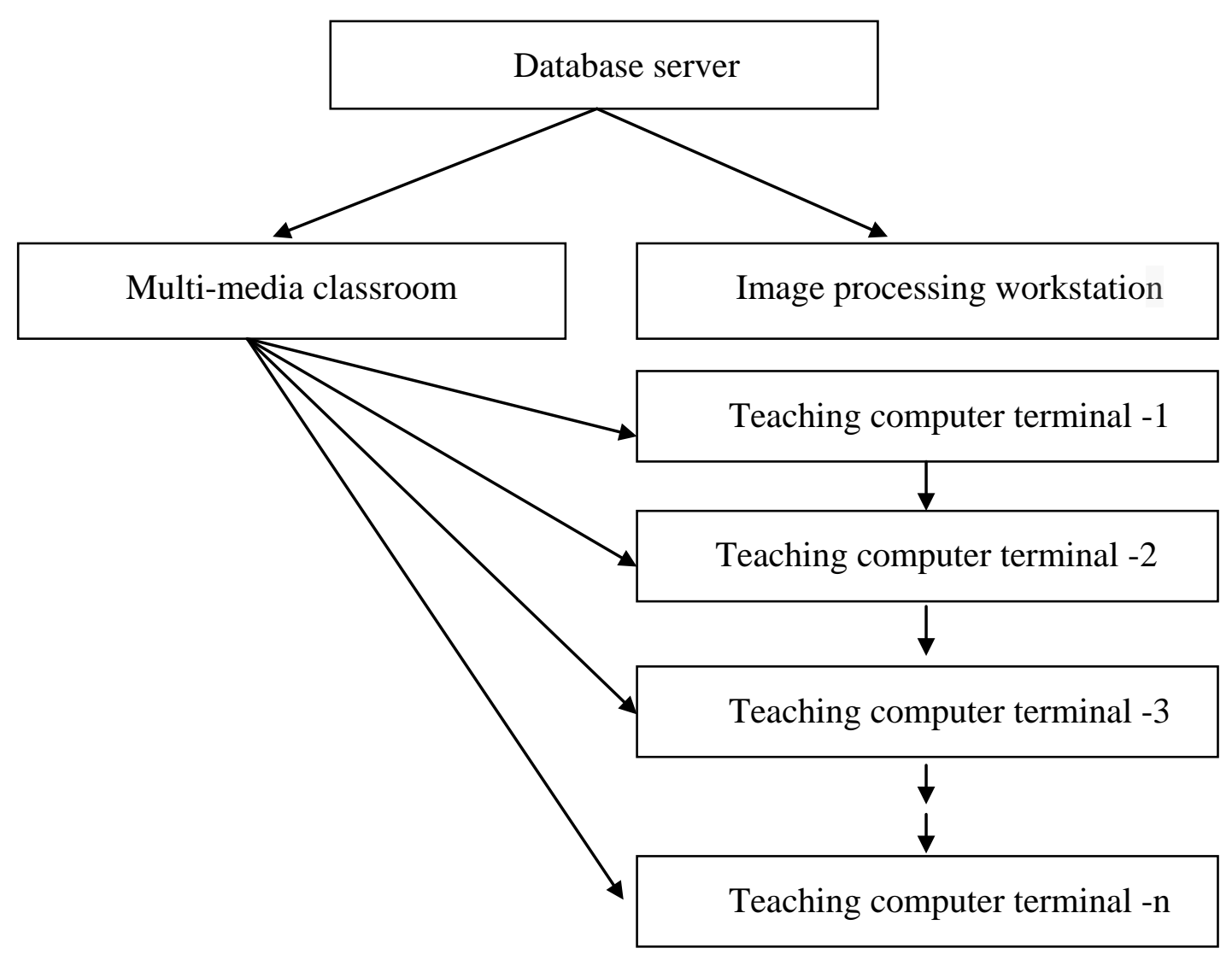

Figure 1 Configuration of the PACS case-based teaching system 


\subsection{PACS Operation and Teaching Methods}

In the experimental class, teachers should use the case based PACS teaching system to teach students. Within the server of the case based PACS teaching system, A lots of typical cases were stored. The teachers can tell students how to use the image post-processing methods to enhance students' understanding of the PACS system. Through the computer operation, students can use the query function to find the relevant images and access to the required images, the operation is convenient and save the time.

\section{Results}

\subsection{Improved the Learning Enthusiasm of the Students}

The case based PACS teaching system includes cases underwent CT or MR examination, which was more than 1000 DICOM format cases and cover the typical and rare performance of common diseases in human system and some rare diseases imaging performance with highly quality. Teachers shorten the preparing time greatly through PACS case-based teaching system and enrich the teaching methods. At the teachers' symposium, the case based PACS teaching system got highly evaluation from the teachers.

Students in the laboratory learn to study by themselves through case based PACS teaching system, reflecting the "multi-clinical practice, repeated clinical practice," they can closely observe the high-quality images consist with the actual clinical work, and through PACS post-processing function to find out their interest cases and signs. Students can learn using images, reports and books, which greatly improve learning efficiency. Students can get post-processing images, such as three-dimensional reconstruction images, multi-directional observation of lesions and adjacent structures, to help the lesion's accurate positioning. At the same time, the original image, the postprocessing images can be display on the same screen by split-screen, while observing a variety of information to help the diagnosis and differential diagnosis lesions, increased students' understanding of theoretical knowledge and mastery of learning interest.

\subsection{Improved the Evaluation of Teaching Satisfaction of the Students}

Through opening the case teaching system based on the PACS, the problem of less experimental time and the lack of rational knowledge of imaging problems in the past have been solved. Students' satisfaction about the teaching has been improved, and the rate of students' evaluation of teaching and assessment has reach $100 \%$ for four consecutive years.

\section{Discussion}

Experimental training plays an important role in the course of teaching the medical diagnostic imaging. It is also an important link for students to consolidate and review theory knowledge and improve clinical practice ability. It is also a key link for students to get used to practical clinical work from classroom. The use of PACS teaching system is to provide a better mean for learning of the medical diagnostic imaging, its enormous clinical cases, high-quality images, powerful postprocessing functions and the method of operation similar to clinical practice in medical diagnostic imaging teaching play an important role. Case based PACS teaching system, greatly increases the student's learning time, while enriching the learning content.

Medical diagnostic imaging is a morphology discipline, so the key of its teaching materials is the medical images. PACS case-based teaching system maintained the original information of the case, the images have high definition, high resolution and true color, and the images can be used to produce the 3D (three-dimensional) reconstruction image, which can make images more perfect [3]. High-quality images of the full range mobilize students's multi-sensory system, so that students get access to significantly increased knowledge. Moreover, students can be familiar with the professional skills and improve their learning efficiency [4-7]. 
Case based PACS teaching system can store a large number of teaching cases in the form of digital images in the server. Through the computer operation, students can use the query function to find the relevant images and access to the required images, the operation is convenient and save the time. Through opening the laboratory of case based PACS teaching system, students can use their spare time for the self-learning, which ensure that each student can see the abnormal changes in the pictures. Through post-processing functions such as regulate window, regulate brightness, measure size and angle to handle pictures, the students can read the images with highest quality and the most complete form [8]. So, the system can enhances the students' learning confidence, improves the efficiency of the theoretical and experimental courses and the students' enthusiasm for learning, which achieves the good teaching effect $[9,10]$. When the traditional teaching model has been unable to adequately meet the teaching needs, case based PACS teaching system achieve the digitization, which has become a major milestone in the reform.

When the students studied in the laboratory, they can use the PACS teaching system for selflearning, and they can read images together and discuss clinical cases. If they have questions, they can search the answer online, or write down the problems to ask the teachers. In the learning process, the students read the images of the cases firstly, combined with the clinical data of the cases and the laboratory examination of its imaging performance analysis, then compared to the attached case report, which promoted students understanding of imaging rationality, and developing the student's abilities of discovering, analysing and solving the clinical problem. In the process of reading and post-processing the medical images, the students deepen the understanding of the basic concepts such as window-technique, CT value, etc. Such process help students to understand the occurrence and development of diseases, the memory process, train students' self-learning, and cultivate the ability to read medical images and think independently [11].

Through the case based PACS teaching system, teachers can log records and recognize the students learning about the situation, points of interest and other information, which help teachers in the theoretical lessons and experimental teaching targeted teaching. The processes reflect the teaching idea of "Student - centered".

In short, development and application of the case based PACS teaching system in the medical diagnostic imaging has become a new era of teaching. Opening laboratory of case based PACS teaching system helps students to learn medical diagnostic imaging into a new impetus and makes medical diagnostic imaging teaching more suited to the development of modern medical digital education, which lays the foundation for training qualified medical imaging undergraduate.

\section{Acknowledgements}

This work was supported by a grant from the Foundation of Chinese Medical Association Medical Education Branch (2016B-YJS031), Foundation of Higher medical education research center of Shandong Province (YJKT201514), Foundation of Postgraduate education innovation program of Shandong Province (SDYY15119), Foundation of weifang medical university (2014Z0301).

\section{References}

[1]Wang B, Zhang SZ, Dong P, et al. Application of PACS teaching system in medical imaging teaching. Chinese Journal of Health Information and Management 2006,3(2):69-70.

[2]Liang XQ, Zhou YY, Chen Q. Advantages of PACS in image teaching. Modern Medical Imagelogy 2010, 24(10):54-55.

[3]Chen SS, Huang MH. Clinical application and experience of PACS system. Journal of Naval General Hospital 2005,18(1):54-55.

[4]Long JF, Jiao ZC, Dong P, et al. The research of PBL independent learning model based on PACS on promoting graduate students' professional knowledge learning. China Higher Medical Education 2013, 2: 130-131. 
[5]Wang B, Zhang SZ, Dong P, et al. The discussion of the criteria on the record of massive increase in digital image. J Pract Radiol 2007, 23(7):1-2.

[6]Wang B, Dong P, Zhang SZ, et al. The application values of the PACS in teaching medical imaging in medical college. Researches in Medical Education 2008, 7(6): 665-666.

[7]Zou YF, Hong XN, Zhou XB. Application of PACS system in medical imaging teaching. Modern Medical Imagelogy 2009, 18(2):124-125.

[8]Duan G, Chen WG, Huang XH, et al. The application of PACS system in the teaching of image diagnosis. Journal of Branch Campus of the First Military Medical University 2003, 26(1):19-20.

[9]Dong P, GAO ZQ, LI YW, et al. The Construction of Medical Imaging Network Teaching System with the PACS in university. ICSAI, 2012, p1023-1025.

[10]Dong P, GAO ZQ, LI YW, et al. The Construction of Medical Imaging Network Teaching System with the PACS in university. ICSAI, 2012, p1023-1025.

[11]Hu J, Ding SY, Li HT, et al. The application of PACS in imaging teaching. Journal of Regional Anatomy and Operative Surgery 2007, 16 (1):56-56. 